\title{
Segmentation of Short Axis Cardiac MR Images using PCA with Active Appearance Model
}

\author{
${ }^{1}$ Radhika Sourabh Inamdar, ${ }^{2}$ Dipali Ramdasi \\ ${ }^{1,2}$ Instrumentation and control Cummins College of Engineering For Women, Pune, India
}

\begin{abstract}
We describe the method for segmentation of Left Ventricle (LV) in short axis cardiac MR Images in order to visibly identify the $L V$, the endocardium and the epicardium. The interpretation and evaluation of images obtained from Magnetic Resonance Imaging (MRI) is not a trivial task and also it is very timeconsuming if done completely manually. This is especially the case for higher dimensional image data such as volumetric and/or time-dependent data such as the Cardiac Images. The objective of this study was show clearly the LV in particular so that any deviation from the standard dimensions in terms of shape, size or texture, can be unmistakably identified. Early diagnosis of heart abnormalities can potentially decrease the risk of heart attacks as well as benefit people who have just undergone Heart Transplant Surgery. For segmentation of the Cardiac MRI, Principal Component Analysis (PCA) is used in the Active Appearance Model (AAM) building process. The extraction of statistical features with PCA can be regarded as a projection of high-dimensional data vectors into a space of much lower dimensionality. The process of building a computerized model is equated with making the computer learn possible appearances of an object. The basis for such a learning process is the training set which is prepared from the data obtained from a reputed hospital in Pune.

Keywords:Magnetic Resonance Imaging(MRI), Left Ventricle(LV), Principal Component Analysis(PCA), Active Appearance Model(AAM).
\end{abstract}

\section{Introduction}

According to the Organ Procurement and Transplantation Network (OPTN), out of approximately 4000 heart transplants performed every year in the United States, 543 patients die in the first year, only $78 \%$ survive 3 years after the transplant and $72 \%$ after 5 years. Most of these deaths are due to rejection of the transplanted organs. To prevent rejection, patients are given immuno-suppressants, which suppress the immune system to enable acceptance of the newly transplanted organ. However, excess of these drugs destroy the patient's immune system making them susceptible to numerous diseases. These patients are monitored closely by doctors to adjust the dosage of immuno-suppressants given to them. One method of doing this is by biopsy, which involves taking a small piece of the heart muscle and inspecting it under the microscope for damaged cells. This procedure is highly invasive and prone to sampling errors. MRI can be used to effectively monitor any functional abnormality of the heart in a non-invasive manner. Segmentation of the cardiac MRI images into epicardium and ventricles helps to detect any deformities and thus could indicate the on-setting of rejection. This segmentation can be performed manually by experts, but it is a time consuming process, and may delay the detection of rejection at a crucial stage. The manually performed segmentation also suffers the problem of inconsistency. Hence, efforts have been made to make this segmentation procedure completely automatic. $[1,2]$

The heart is a muscular organ of the circulatory system that constantly pumps blood throughout the body. As seen in figure 1, the heart has four separate chambers. The upper chambers are called the left and right atria, and the lower chambers are called the left and right ventricles. The septum separates the right and left sides of the heart. The endocardium is the inner surface of the myocardium (the heart muscle tissue) and the epicardium is the outer surface of the myocardium. The muscular tissues that attach to the lower portion of the interior wall of the ventricles are the papillary muscles. The periodic motion of the walls of the heart chambers during one heartbeat is referred to as one cardiac cycle. One heartbeat consists of two phases, namely systole and diastole. The rhythmic contraction of the ventricles, by which blood is driven through the aorta and pulmonary artery is the systole. Diastole is the normal rhythmically occurring relaxation and dilatation of the ventricles, during which they fill with blood. The right ventricle delivers blood to the lungs. The left ventricle delivers blood to the rest of the body. So the left ventricular wall is thicker. This fact is often used for distinguishing between the left and right ventricles. Here, we study the MRI images of the transverse section of the heart and segment them into epicardium, right ventricle, and left ventricle. [1,2]

Magnetic Resonance Imaging (MRI) is a method of creating images of the inside of opaque organs in living organisms. When the object to be imaged is placed in a powerful uniform magnetic field, the spins of the hydrogen nuclei with non-zero spin numbers, within the tissue, all align in one of two opposite directions: parallel to the magnetic field or three orthogonal image gradients are applied, to selectively image the different voxels (3-D pixels). They are the slice selection gradient, the phase encoding gradient, and the frequency 
encoding gradient. As the high energy nuclei relax and realign, they emit energy, which is recorded to provide information about their environment. The primary advantage of MRI is the high contrast resolution between different soft-tissue types. [1]

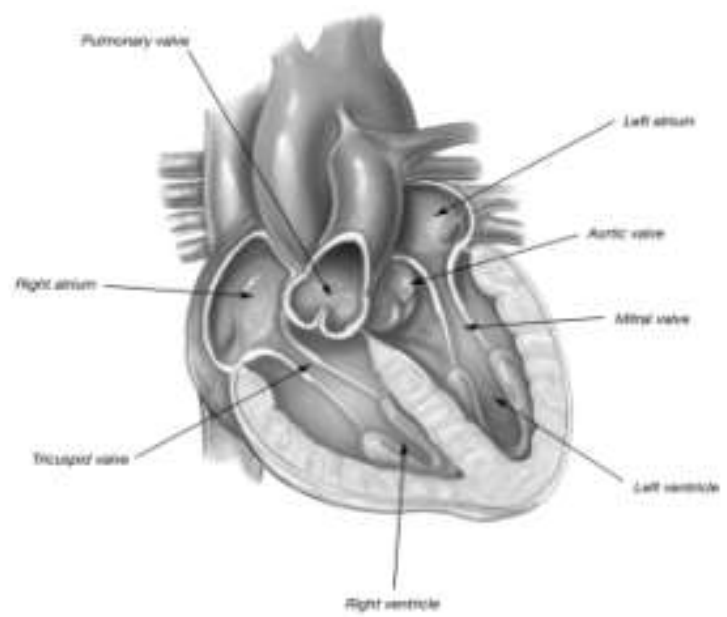

Fig. 1 Anatomy of the Human Heart [16]

\section{Short Axis cardiac Image}

The complexity of segmenting heart chambers and myocardium mainly relies on heart anatomy and MRI acquisition specificity. The LV function consists in pumping the oxygenated blood to the aorta and consequently to the systemic circuit. The LV cavity has a well known shape of ellipsoid [Fig 1] and is surrounded by the myocardium, whose normal values for thickness range from 6 to $16 \mathrm{~mm}$ [2]. On the contrary, the RV has a complex crescent shape. It also faces lower pressure to eject blood to the lungs and is thus three to six times thinner than the LV, reaching the limit of MRI spatial resolution. For those reasons, and because its function is less vital than the LVs, most research effort has focused on the LV, even though MRI has proven to provide an accurate quantification of RV mass. The standard imaging plane is perpendicular to the long (apexbase) axis and called short axis plane [Fig 2]. Imaging of the heart in MRI covers the whole organ with about 8 10 short-axis slices, distance between two adjacent slices ranging from 10 to $20 \mathrm{~mm}$. As the heart is a moving organ, images are acquired throughout the cardiac cycle, thanks to the synchronization of MR acquisition to the ECG signal and the use of cine (i.e. dynamic) MR sequences. In full-size images, the ventricles cover quite a small surface in cardiac MR images and processing is usually restricted to a smaller region of interest (ROI). About thirty phases (or images) can be obtained during one cardiac cycle with the currently available equipment, yielding a temporal resolution of about $30 \mathrm{~ms}$. The number of phases decreases proportionally to the heart rate. A single examination can thus be made up of 250 images. [2]

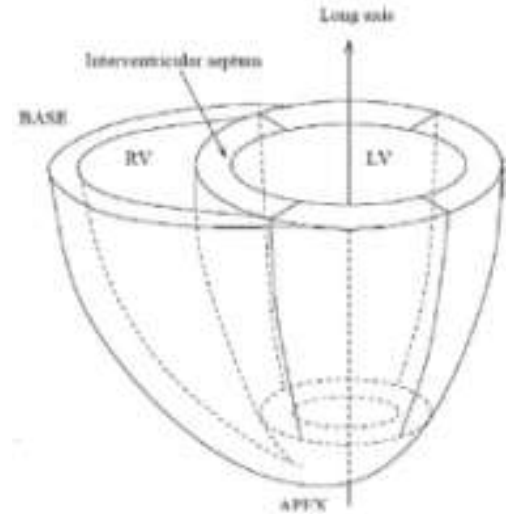

Fig. 2 LV and RV Geometry [2]

\section{Materials and Methods}

Numerous segmentation methods have been used to study the heart and diagnose different heart ailments effectively. 


\section{A. Categorization of Short-Axis Cardiac Image Segmentation}

A common categorization for medical image segmentation includes thresholding, edge-based and region-based approaches, pixel-based classification, and atlas-guided approaches. Image-driven techniques, such as thresholding, region-based or edge-based techniques, or else pixel classification, offer a limited framework for strong prior incorporation [2, 5, 7, 810,11$]$. Deformable models, including snakes and their variants, on the contrary, offer a great, versatile framework for using either weak or strong prior. They can include anatomical information, as well as high level information, in the so-called shape prior based segmentation framework, or through active shape and appearance models (ASM/AAM). At last, atlas guided segmentation also make use of a set of manually segmented images. Considering both the usual segmentation method categorization and the level of external information, we propose the following two main categories [2]:

- Segmentation based on no or weak prior, that includes image based and pixel classification based methods, as well as deformable models;

- Segmentation based on strong prior, that includes shape prior based deformable models, active shape and appearance models, and atlas based methods.

\section{B. Segmentation with Strong Prior}

As seen by the growing literature on this matter, automatic organ segmentation can benefit from the use of a statistical model regarding shape and/or gray levels, to increase its robustness and accuracy. This especially applies if the nature of the shape does not change much from an individual to another, which is typically the case for the heart [2]. Statistical-model based segmentation techniques comprise three steps: [2]

1. Spatial (and temporal if required) alignment of manually segmented contours or images is of utmost importance to compensate for differences in ventricle position and size, and can be very difficult. One subject in the database is arbitrarily chosen as reference. Every other subject is affinely registered on the reference, and an average shape is computed [2]. This procedure can be performed iteratively by replacing the reference subject with the mean model. When segmentation is computed over the complete cardiac cycle, temporal alignment is needed as well: the number of phases of each individual must be aligned on the number of phases of the reference and thus requires resampling and interpolation.

2. Model construction generally implies the computation of an average shape or image and the modeling of variability present in the training images and contours. This latter can be made via the widely used principal component analysis (PCA), when point correspondences between contour points are available, or else via variance computation or probability density modeling. The PCA provides the eigen-value decomposition of the shape set covariance matrix, yielding principal eigenvectors (or components), that account for as much of the variability in the data as possible [2]. Each instance of a new shape can thus be described by the mean shape and a weighted linear combination of eigenvectors.

3. The use of the model for segmentation specifies how the average model is applied to fit the contours in a new image, while taking into account the variability present in the training set.

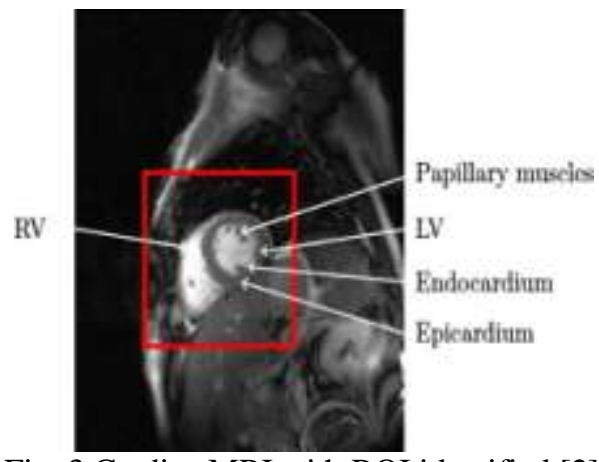

Fig. 3 Cardiac MRI with ROI identified [2]

\section{Active Appearance Model (AAM)}

The Active Shape Model (ASM) consist of a statistical shape model, called Point Distribution Model (PDM), obtained by a PCA on the set of aligned shapes, and a method for searching the model in an image. PCA is used in order to reduce the amount of data that needs to be handled. Segmentation is performed by placing the model on the image, and iteratively estimating rotation, translation and scaling parameters using least square estimation, while constraining the weights of the instance shape to stay within suitable limits for similar shapes [5, 6, 7, 9 10,11]. ASM have been extended to gray level modeling, yielding Active appearance models (AAM) that represents both the shape and texture variability seen in a training set [19]. This technique ensures to have a realistic solution, since only shapes similar to the training set are allowed. [Fig. 3] 
The first type of information included in an AAM normally is referred to as shape. Very important for the concept of shape is that shape has to be independent of rotation, scaling and translation. In computer vision and pattern recognition the concept of landmarks was introduced to effectively represent shapes. Identifying the shape of an object then means to identify distinctive points the landmark points in the geometrical appearance of this object. [19]

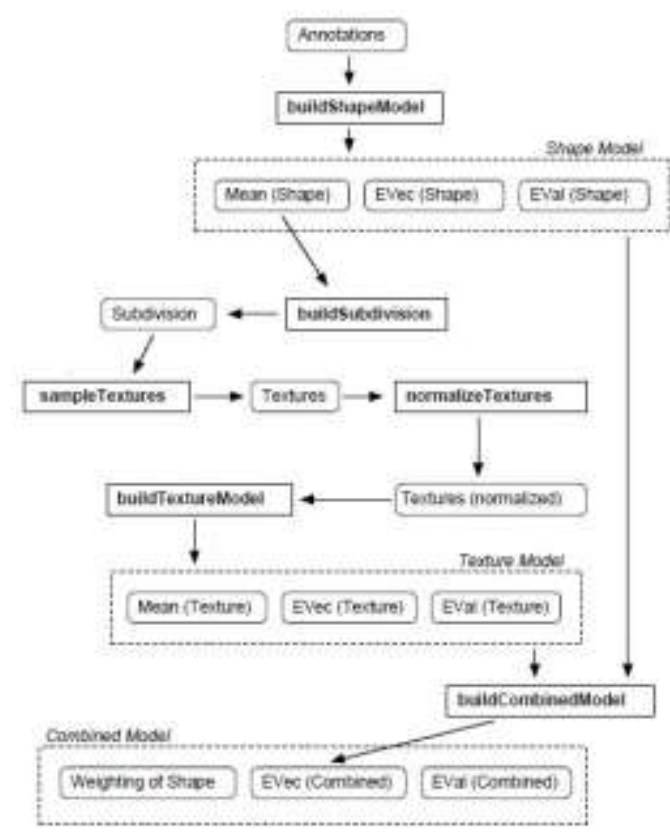

Fig. 4 The AAM Model

The data analyzed for building a texture model is usually represented in form of simple gray values. The first step in building a texture model is to find out which gray values are important. This has to be done since the complete image data usually does not only include gray values of the object for which we want to build the model. Also texture information resides in the image which comes from things which are distinct from our object of interest.
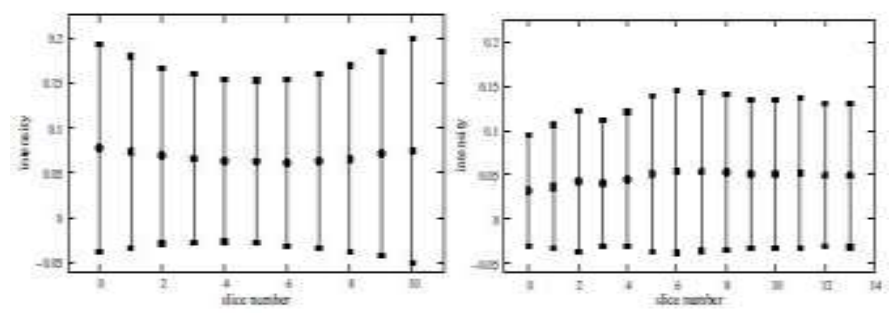

Fig. 5 The mean values and standard deviations of texture in individual slices of two data sets [19]

The first stage is forming the Shape Model for the cardiac images followed by forming the texture model for the respective images. Now the combined model is obtained and in this manner the training data set is prepared. The segmented image looks as shown in Fig. 5. The process of obtaining the various models is shown in Fig. 6.

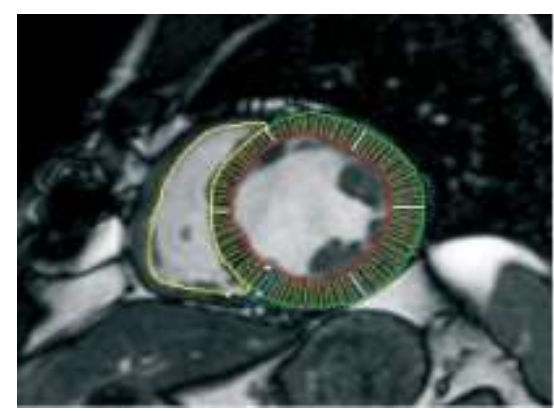

Fig. 5 Endo and Epi-cardial contours of the Ventricles are shown [16] 


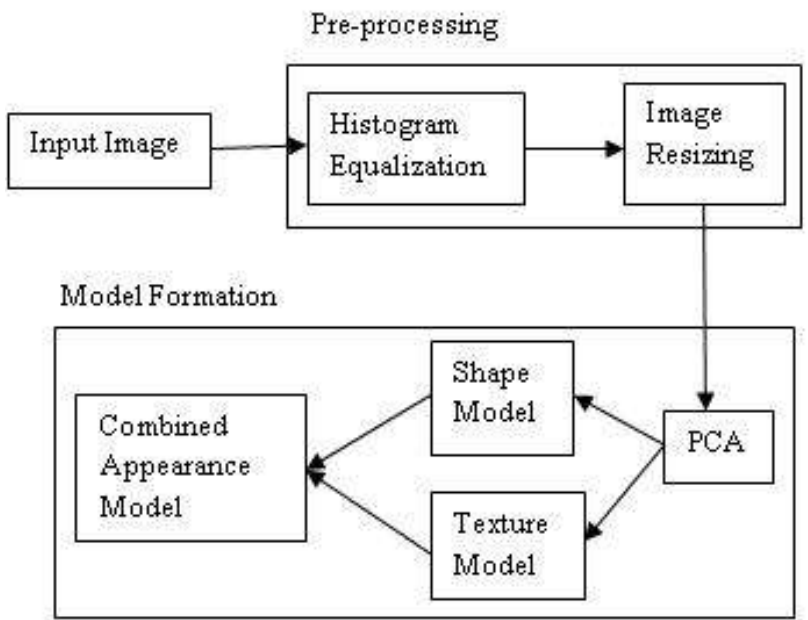

Fig. 6 Block Diagram for forming the Active Appearance Model (AAM)

After the models for various cardiac shape variations is identified, the new images are then compared to the training set and the appropriate match is identified as shown in Fig. 7.

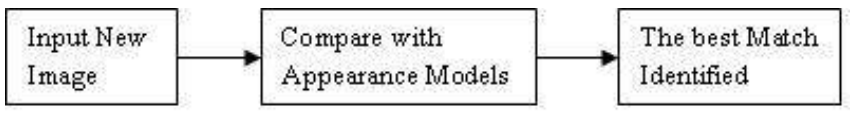

Fig. 7 Processing Stage

Although the AAM technique demonstrated segmentation reliability, there are always concerns using a modelbased method built upon a finite set of training data to correctly handle often grossly abnormal patient data. One solution to this problem is to utilize larger training sets to cover all representative pathologies. $[5,6,7,8,9,11$, 22]

\section{Discussion}

Cardiac Ailments can lead to an increased risk of heart failure. This is the case especially in times of heart transplant, when there are a lot of chances of cardiac failure as the patient's body might reject the transplanted heart. By constructing an AAM for the various heart ailments it is possible to a large extent to identify small variations in the cardiac shape or texture at the earliest and hence avoid a possibly fatal heart attack to a person or diagnose a heart disease at the earliest. Also by working with the doctors it is possible to make the AAM models of most heart ailments and eventually diagnose most heart disorders.

\section{Conclusion}

Thus this paper proposes an approach for identifying the cardiac ailments automatically. We have outlined the importance of fast and automatic detection of heart diseases. We shortly reviewed the anatomical background and outlined special properties of cardiac MRI data. The purpose of this study was to obtain a possible means of early detection of cardiac failure by MRI examination, which is simple, and affordable by public as well as cost effective. In this work we first determine region of interest based on the position of the heart inside the chest cavity. Since we need to distinguish the heart from other object, image enhancement is done. For developing the various models for different ailments PCA is used in forming an AAM and then the various models are compared with the new images in order to classify them under the appropriate heart disease.

\section{Acknowledgment}

The authors take this opportunity to express deep sense of gratitude to the principal, Dr. Mrs. Madhuri Khambate and Prof. A.D. Gaikwad for their support.

\section{References}

[1] Neeti Gore. "Image Segmentation Combining Level Sets and Principal Component Analysis" A Thesis. Department of Electrical and Computer Engineering, Carnegie Mellon University.

[2] Caroline Petitjean, Jean-Nicolas Dacher. "A review of segmentation methods in short axis cardiac MR images" Science Direct Medical Image Analysis.

[3] Andy Tsai*, Anthony Yezzi, Jr., William Wells, Clare Tempany, Dewey Tucker, Ayres Fan, W. Eric Grimson, and Alan Willsky. "A Shape-Based Approach to the Segmentation of Medical Imagery Using Level Sets" in IEEE Transactions on Medical Imaging, Vol. 22, No. 2, pp. 137-154 February 2003 
[4] Avan Suinesiaputra, Alejandro F. Frangi, A. M. Kaandorp, Hildo J. Lamb, Jeroen J. Bax, Johan H. C. Reiber, and Boudewijn P. F. Lelieveldt. "Automated Detection of Regional Wall Motion Abnormalities Based on a Statistical Model Applied to Multislice Short-Axis Cardiac MR Images” in IEEE Transactions on Medical Imaging,, Vol. 28, No. 4, pp. 595-607, April 2009

[5] Timothy F. Cootes and Christopher J. Taylor. A mixture model for representing shape variation. Image and Vision Computing, Vol. 17, No.8, pp. 567-573, 1999.

[6] Timothy F. Cootes, Gareth J. Edwards, and Christopher J. Taylor. A comparative evaluation of active appearance model algorithms. In Proceedings of the British Machine Vision Conference (BMVC), vol. 2, pp 484-498, 1998

[7] T. F. Cootes, G. J. Edwards, and C. J. Taylor. Active appearance models. IEEE Transactions on Pattern Analysis and Machine Intelligence, Vol.23, No(6): pp 681-685, June 2001

[8] G. J. Edwards, C. J. Taylor, and T. F. Cootes. Interpreting face images using active appearance models. In Proc. International Conference on Automatic Face and Gesture Recognition, pp 300-305, June 1998.

[9] T. Cootes, G. Edwards, and C. Taylor. Active appearance models. In Proceedings of the European Conference on Computer Vision, volume 2, pages 484-498, 1998.

[10] G. J. Edwards. Learning to Identify Faces in Images and Video Sequences. PhD thesis, University of Manchester, Division of Imaging Science and Biomedical Engineering, 1999.

[11] T. F. Cootes. Statistical models of appearance for computer vision. Online technical report available from http://www.isbe.man.ac.uk/?bim/refs.html, Sept. 2001.

[12] Yun Zhu, Albert J. Sinusas, and James S. Duncan. "Integrated Segmentation and Motion Analysis of Cardiac MR Images Using a Subject-Specific Dynamical Model” in IEEE Transactions on Medical Imaging,, Vol. 30, No. 4, pp. 595 -607 April 2009

[13] S. Roujol1, J. Benois Pineau, B. Denis de Senneville, B. Quesson, M. Ries, C. Moonen. "Real Time Constrained Motion Estimation for ECG-Gated Cardiac MRI" in Proceedings of 2010 IEEE 17th International Conference on Image Processing September 26-29, pp. 557-560, 2010, Hong Kong

[14] M. U“zu“mcu“, A.F. Frangi, J. Reiber, and B. Lelieveldt. "ICA vs. PCA Active Appearance Models: Application to Cardiac MR Segmentation" in R.E. Ellis and T.M. Peters (Eds.): MICCAI 2003, LNCS 2878, pp. 451-458, 2003 Springer-Verlag Berlin Heidelberg 2003

[15] Aapo Hyvrinen and Erkki Oja. "Independent Component Analysis: Algorithms and Applications" in Finland Neural Networks, pp. 411-430, 2000

[16] Prof. dr. A. de Roos, Ir. R.J. van der Geest, Dr. A.F. Frangi. "Constrained Segmentation of Cardiac MR Image Sequences" in Universal Press, Veenendaal, The Netherlands ISBN 978-90-9022186-1 2007

[17] Weng, J., Singh, A., Chiu, M., 1997. Learning-based ventricle detection from cardiac MR and CT images. IEEE Trans. Med. Imag. Vol.16, No.4, pp 378-391.

[18] Steven C. Mitchell, Boudewijn P. F. Lelieveldt, Rob J. van der Geest, Johan G.Bosch, Johan H. C. Reiber, and Milan Sonka. Multistage hybrid active appearance model matching: Segmentation of left and right ventricles in cardiac MR images. IEEE Transactions on Medical Imaging, 20(5):415-423, 2001.

[19] Steven C. Mitchell, Johan G. Bosch, P. F. Lelieveldt, Rob J. van der Geest, Johan H. C. Reiber, and Milan Sonka. 3D active appearance models: Segmentation of cardiac MR and ultrasound images. IEEE Transactions on Medical Imaging, 21(9):pp 1167$1178,2002$.

[20] A. F. Frangi, W. J. Niessen, and M. A. Viergever, "Three-dimensional modeling for functional analysis of cardiac images: A review,” IEEE Trans. Med. Imag., vol. 20, pp. 2-25, Jan. 2001.

[21] N. Duta and M. Sonka, "Segmentation and interpretation of MR brain images: An improved active shape model," IEEE Trans. Med. Imag., vol.17, pp. 1049-1062, Dec. 1998.

[22] Dr.techn. Jiri Hladuvka, 3D Active Appearance Models for Segmentation of Cardiac MRI Data. A Thesis

[23] Jonathon Shlens, A Tutorial on Principal Component Analysis, Center for Neural Science, New York University New York City, NY 10003-6603 and Systems Neurobiology Laboratory, Salk Insitute for Biological Studies La Jolla, CA 92037 (Dated: April 22 , 2009; Version 3.01)

[24] Sebastian Zambal, Jǐr'1 Hlad $^{\circ}$ uvka, and Katja B“uhler. VRV is Research Center for Virtual Reality and Visualization, Donau-CityStrasse 1, 1220 Vienna, Austria. http://www.vrvis.at

[25] Rafael C. Gonzalez, Richard E. Woods, "Digital Image Processing”, 7th ed. Prentice Hall, 2002, pp 92-100.

[26] Uday Kurkure, Amol Pednekar, Raja Muthupillai, Scott D. Flamm, and Ioannis A. Kakadiaris. "Localization and Segmentation of Left Ventricle in Cardiac Cine-MR Images” in IEEE Transactions on Biomedical Engineering, Vol. 56, no. 5, pp. 1360 - 1370 May 2009 